\title{
Blockchain enabled technology platform for enhancing supply chain financing for SME's
}

\author{
Karthik M' $\mathbf{1}^{\text {, Dr. Shilpa Parkhi }}{ }^{2}$ \\ ${ }^{1}$ Symbiosis Institute of Operations Management, Nashik, Symbiosis International (Deemed University), Pune, India \\ ${ }^{2}$ Professor and Deputy Director, Symbiosis Institute of Operations Management, Nashik, Symbiosis , International (Deemed \\ University), Pune, India.
}

\begin{abstract}
After the development and inception of cryptocurrencies, Blockchain has found much usefulness in many domains. The Blockchain technology offers several advantages like decentralization, traceability, anonymity, cryptography, collective maintenance and immutability. The possible disruption and prospective capabilities of the Blockchain technology has been extensively utilized in the Finance domain. There has been extensive exploration on the integration and application of Blockchain technology in the domain of supply chain and supply chain finance as well. But, a detail framework of application is seldom mentioned. In this research, we have studied and analyzed the possible synchronization between Blockchain technology and supply chain finance. This study also presents a conceptual framework based on Blockchain technology to enhance the efficiency of supply chain financing for small and medium scale enterprises. Finally, this study proposes three supply chain finance models and their operation processes on a Blockchain Platform.
\end{abstract}

Keywords:

Supply chain finance, smart contract, decentralization, Blockchain technology, traceability, immutability, financial institutions.

Article Received: 10 August 2020, Revised: 25 October 2020, Accepted: 18 November 2020

\section{Introduction}

The popularity of Bitcoin has made Blockchain a very attractive and interesting, useful technology. Nakomoto conceptualized a technology for cryptocurrencies called Blockchain to store and share Bitcoin transactions. Blockchain technology instead of just being used for sharing transactions, it can be used to share any type of data including the asset related data, demand data, invoices, inventory data that are useful to the supply chain parties. Using Blockchain technology distribution information sharing can be achieved without the need for any intermediary institutes or personnel. Blockchain technology can be used by parties in the supply chain network for sharing data directly with other firms in their supply chain to reduce the existing information asymmetry. The signaling can be achieved by facilitating blockchain to store and share information. Using Smart Contracts, private and consortium blockchain can also regulate the participants of who can be a part of the network and who can access what data. Moreover, the immutable nature of the information stored in the blockchain as well as the features of time stamps and encryption makes it a trustworthy platform to share information between supply chain partners. Hence blockchain technology can be leveraged for network signaling in the SCF context as it can be used for facilitating secure information sharing to reduce information asymmetry.

Blockchain has been coupled with diverse technology and fields like Internet of Things (IoT) , security systems, public services and regulatory services ( Sharples and Domingue, 2016). Walmart led syndicate of companies, with IBM have identified how global food supply chains can leverage Blockchain technology. They also conducted a pilot study to track pork meat in China.

Across the world, the shortage of working capital has been a major pain point for small and medium-sized enterprises (SMEs). There exists a finance gap for these SMEs (Mina et al., 2013). Though there are opportunities which are potentially profitable, they are deficient of funds to leverage them. The existing or available financial resources are usually not sufficient to strike a balance between survival and growth strategies of these organizations. In order to strike a balance, the owners or managers become dependent on family, friends or acquaintances. If the gathered funds are not sufficient they become 
dependent on external sources of financing from venture capitalists, banks or other financial institutes. The gathered internal funds will suffice the capital required during the early stages of operations. But, in order to keep the business growing external finance is required(Vos et al., 2007). However, information silos or the lack of transparency about the SME's operations affect the perceptions and decisions of the financiers. Casey \& O'Toole, (2014), suggests they need reliable information for assessment to evaluate the quality of the SMEs.

Song and Zhang, 2018 has shown how traditional methods of financing and signaling which includes securing mortgages or collateral, guarantees for credit or financial statements were not effective. According to (Serrasqueiro, 2011) one of the challenges is that the SMEs deficient collaterals or the presence of unorganized assets which make the financiers search for relevant information to perform credit evaluation. In this situation, the financiers who are highly dependent on the signals comprising objective information would not consider certain SMEs who transmit verbal subjective signals like introducing new products, make press releases, hire trailblazers, etc. (Steigenberger and Wihelm, 2018). Another challenge would be to establish a rational between the intention of borrowing of the borrower who emits the signal and the need for information of the lender who receives the signal. Rather than an intentional signal from one side, if integrative signals from all the members in the network, consistency can be achieved. According to Plummer et al., (2016) and $\mathrm{Wu}$ et al., (2017), to avoid the lack of stability in traditional information transmission by signaling, these signals from supply chain networks can have restricted or no control by the SME signalers (Posthuma et al.,2018). Financiers who are not in the supply chain will face difficulties in gathering these signals.

There have been 3 constraints with Supply chain funding: 1) Confirming transaction authenticity, 2) Enterprise credit transfer, and 3) High cost of Risk Control.

Blockchain technology finds well suited application to supply chain finance business scenarios since the transaction frequencies are relatively low, and there is a need for trust building at multiple dependent nodes. Advantages that Blockchain technology offers supply chain finance are: 1) Can verify transaction authenticity. The features of traceability, auditability and time stamps will help the financial institutions to validate transaction background's authenticity.

Due to the sophisticated supply chain network, the credit score transferring to the end of the supply chain was ambiguous. Blockchain technology will enable the credit scores transfer in simple and hassle free manner. Various bills like logistic receipts, acceptance bills, online pledges, recievables can be digitized and registered on the Blockchain platform. 3) The negotiation or execution of smart contract can be enforced, facilitated and verified using Smart Contract (a computer protocol customized to the business process). A smart contract is uploaded onto the Blockchain platform using a programming language. Once the conditions mentioned in the contract are met, the agreement contents are automatically executed which lead to a transaction of property transfer. 4) Ensures data security with features of encryption and immutability. This can solve the problem of bill losses since all the nodes formed after transaction are interconnected. 5) Digitization of assets will help to trace the flow of assets. Blockchain works on the concept of tokenization. In 2018, IBM proposed a plan to Tokenise carbon emission allowance. Similarly, flow of goods information and the subsequent pledges can be recorded on the Blockchain platform.

Low level of digitization and infrastructural constraints of bill market and lack of judicial support are major challenges for large scale application of Blockchain technology in the field of supply chain finance. However, if Blockchain technology is implemented, core enterprises will be motivated to participate,smart contract will solve security and legal issues and the cost of risk for various financial institutions reduces.

After a detailed study and analysis, we propose a Blockchain based platform as a solution to supply chain finance problems.In sync with the business process of supply chain finance (SCF), this study proposes a conceptual framework of Blockchain Technology Platform for Supply chain Financing (BTP-SCF). To illustrate some details three models of SCF business processes on BTP-SCF are given.

The research is structured as follows : Section 2 contains the Literature survey on Blockchain and how it can be applied in the domain of Supply 
chain Finance. Section 3 describes the conceptual framework of BTP-SCF, its scheme of integration, and the node types on the platform. Section 4 illustrates business process of SCF like prepayment financing, Account Receivable receipts and pledged assets financing. Section 5 gives a summary of the entire study and also discusses the scope for future work.

\subsection{The Blockchain}

\section{Literature Survey}

A data base that is continuously growing and stores data in the form of structures which are interconnected to forma a chain called a Blockchain. The blockchain system comprises of a consensus algorithm for recording and updating the data, an encryption which is to protect privacy of users and secure the data, and smart contract to make sure the contracts are implemented. When Nakamoto( 2018) introduced his first white paper on blockchain, marked the inception of an extraordinary idea of utility.

Some of the existing literatures have put their main focus on the areas in which the BT can be improved to tackle the it's challenges. Zamani et al., (2018), showed that in past few years, the frequent concerns regarding blockchain's security, resulting in substantial monitory losses. Lin and Liao (2017), implied that given the fact that the bitcoin was the earliest application of blockchain technology, which is a decentralized digital currency, the biggest challenge for the blockchain development and it application is always the matter of security and lack of trust. The leakage of private information and scalability of the technology have been the most common associated problems with the blockchain technology (Li et al., 2020). Particularly, smart contracts have been the prime area of focus (Wang et al., 2018). Zheng et al., (2018), showed that there have been various research and exploration have centered around the innovation in utilization of blockchain technology in various domains, like IoT, Supply Chain Finance, reputation systems and crowd funding. To understand this we can leverage the example where, Saberi et al. (2019) showed how blockchain technology increases the efficiency of logistics management by coupling with IoT technology to provide transparency in data collection and traceability of goods and conditional information. Babich and Hilary (2019) carried out their research in the area of application of blockchain technology in the domain of Operations management. On the basis of utilization of blockchain technology in operations management, they had summarized their research into three categories after studying the disadvantages and advantages that are associated with blockchain. Considering the reciprocity that exists among the manufacturers and the counterfeiters, Pun et al. (2018), showed how Blockchain Technology can be used against counterfeiting of goods. Tian (2016), showed how blockchain and IoT together can be used to improvise the standard and safety related to food products. He also proposed a business process in the context of practical operations applications.

With a combination of blockchain technology coupled with an open manufacturing environment, Li et al. (2018), suggested a framework for cross functional operations. To improve the efficiency of payment settlements in the logistics sector, Álvarez-Díaz et al. (2017), researched on the utilization of smart contracts and suggested a business process to do away with the traditional methods which involves contracts that were made on paper.

\subsection{Supply chain finance (SCF) and application of Blockchain in SCF}

The primary research activities on Supply Chain Finance happened back in the 1970's, when Budin and Eapen, (1970); Haley and Higgins, (1973) began to explore the relationship and coordinated characteristics between trade credit and operations management. Wuttke et al., (2013) showed Supply Chain Finance's main purpose is to optimize cash flow in a supply chain with trade relationship between both upstream and downstream and the endorsements of credit to core enterprises. The features of transaction traceability for the suppliers will negatively impact the transfer of asset opportunity for the borrowers (Burkart et al.,2004). Fabbri and Menichini (2016) and Chod (2017) research gave us an understanding the visibility of transactions will solve the problems related to the replacement of assets. Tang et al. (2018) researched and proved how important it is and also what advantage one gets having certain information in an environment where asymmetry of information exist, by taking into account the source of risk on the basis of supply capacity of the supplier. All the related, studied literatures have shown us that in the business processes of supply chain funding, transparency and 
information symmetry are the key to success. Block chain technology will help the companies in the network to achieve it.

There have been a lot of research conducted by various scholars in the area of blockchain utilization in supply chain finance domain. Hofmann et al. (2017) ascertained all the advantages that the application of blockchain offers to the field of supply chain finance. Chod et al. (2019) showed how blockchain will help cut costs on signaling by offering a window which will make supply chain finance operations very transparent. There are various platforms which are emerging powered by blockchain technology. These aim at improving efficiency of financing and reducing the cost of financing by removing silos and establishing symmetry of information.

Previously, there have been many attempts to stablish blockchain applications in SCF domain. But all these research have only studied the relationship between the two and possible integration theoretically. Our study focusses on the detailed structure of establishing utility of Blockchain technology for business processes of SCF with the help of BTP-SCF platform. This study also describes the conceptual framework of BTP-SCF and its application to three SCF business processes.

\section{Blockchain Technology Platform for Supply}

Chain Financing (BTP-SCF) - A Conceptual Framework

The BTP-SCF is proposed as an enabling platform and integration solution to enhance performance of financing processes and ease the process of obtaining loans for enterprises of small and medium scale. In supply chain financing, there are five main stakeholders involved, namely, loan company, core company, financial service provider, logistic provider (mainly 3PL) and regulatory bodies

\subsection{Choosing the suitable Blockchain}

Based on the degree of decentralization, there are three distinct types of Blockchain namely, Public, Consortium and Private. Based on the business processes involved in supply chain financing, characteristics and degree of information security and transparency required, the suitable type of the Blockchain is selected.

In Public Blockchain anybody or everyone in the network can act as a node and are not dependent on anyone else to gain access to block information. Hence, public Blockchain is absolutely decentralized and every node can process, verify, update and store data on the Blockchain. For any independent organization, Private Blockchain is suitable since they are centralized. In order to facilitate, enable the control and deployment of the centeral organization, only certain nodes with permission in the network can join the Blockchain. One can find partial decentralization in Consortium Blockchain. The process involved in achieving the consensus mechanism is controlled only by certain specific nodes. For executing a transaction or an accounting process only those specific nodes in the network of the chain can participate.

Due to the high accessibility nature of the Public Blockchain, it is not that supportive to privacy protection. Since there area lot of nodes and all the nodes are involved, the transaction speeds are very low. In Private Blockchains, the rights to the consensus mechanism and data management is concentrated which makes the control and management power lie with a single entity. In case of supply chain financing, there is need for high transaction speed as well as a provision for giving specific authorities to certain nodes. This can be achieved by smart contracts on s Consortium Blockchain. Hence for all the business processes in supply chain financing, the BTP-SCF should use a Consortium Blockchain.

\subsection{Types of nodes in BTP-SCF}

The consortium Blockchain is used as the core technology in BTP-SCF. There are five kinds of node types- loan company, core company, financial service provider, logistic provider (mainly 3PL) and regulatory bodies. Every node need to share their enterprise data on to the Blockchain platform. Every node performs their specific duties in the network on this platform. The embedded smart contracts on the plat form ensure the execution and completion of the financial business. The authority to view data can be changed based on the necessities. Any two nodes can interact/ transact point to point. This can enable and ensure information sharing and credit transfer in the network. Refer Figure 1 and Figure 2 to understand the functioning. 


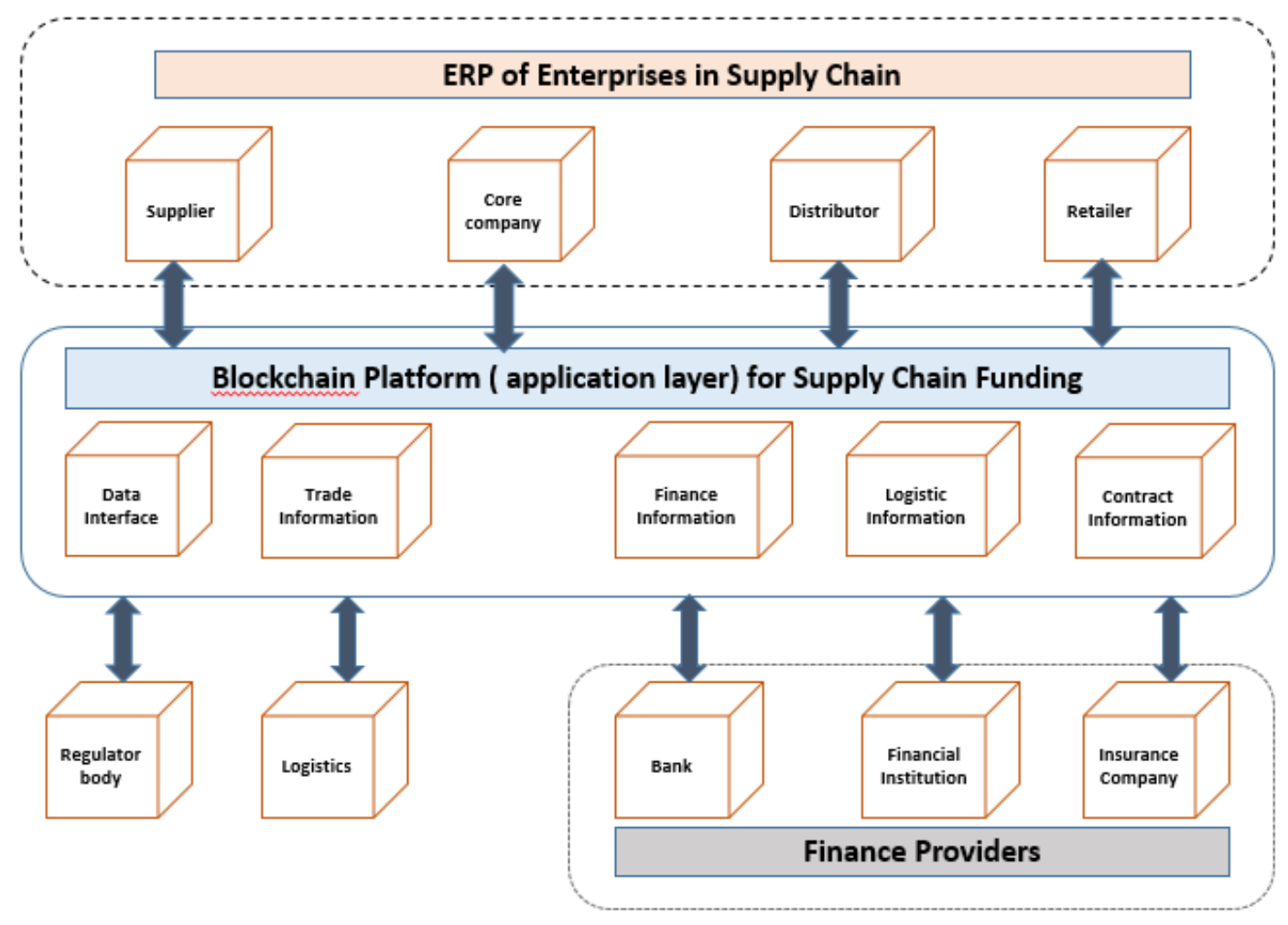

Figure 1: Interaction/ Transaction between the nodes

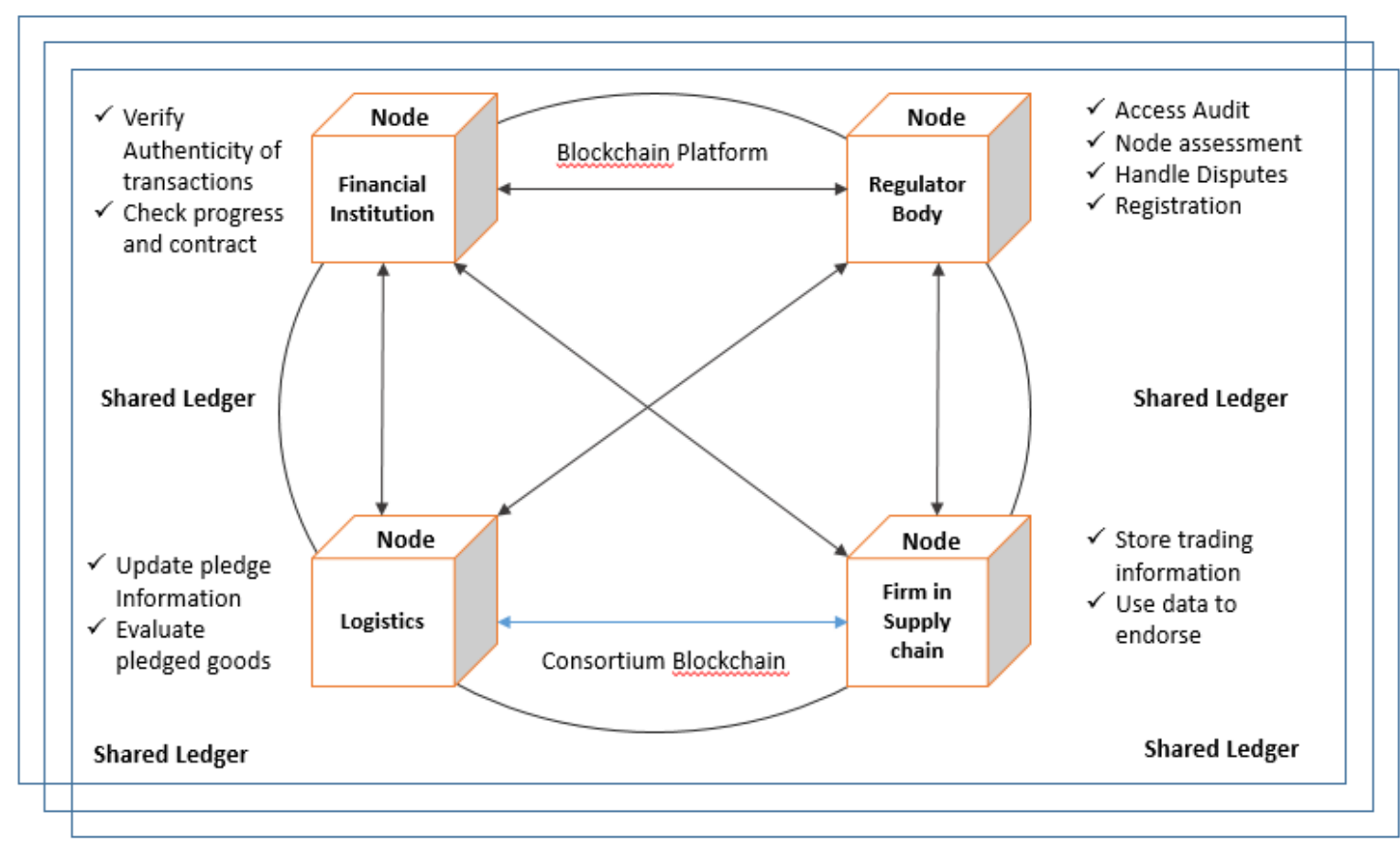

Figure 2: Functional representation and Information flow.

The primary nodes include:

1) Financial Institution: The provider of funds and financial services. These primarily are banks which verify the authenticity of transactions of the loan seeking companies on the Blockchain platform. Whenever a loan application was submitted, banks following traditional model were not able to find reliable transaction data due to asymmetry of information present in the supply chain network as well as the application. Thus for the purpose of validating the submitted financial information and transactions, the bank ( or any financial institution) used to expend a lot of valuable resources like 
time, money and man power. This methodology of credit evaluation is very expensive and one does not get reliable data. However, if BTP-SCF is used, all the transaction including the past trading history of the company are recorded with time stamps, along with the inventory information and order statuses. This data on the Blockchain platform cannot be tampered (immutable) and hence it is highly reliable. By generating a query, all required information pertaining to the loan seeking comoany can be acquired by the bank ( or any financial institution) from BTP-SCF, in order to make a lending decision based on its judgement about the company's reputation. This information will have high credibility and the cost of credit investigation will drastically reduce.

2) Regulatory Body: Government agencies or bureaus like commercial or industrial or tax bureaus which ensure the adherence to government law and orders. They review the new node entries along with the core companies in the consortium blockchain. The evaluate transactions and interactions of the member nodes and also by voting reject applications whose credit standards are not up to the mark and also have certain regulatory discrepancies.

3) Firm in Supply Chain : A small or medium enterprise seeking financial funding. These firms, as well as their core firm's upstream suppliers and downstream retailers are capital constrained in the entire supply chain. Due to unstable operations, high risks and small scale of operations, these firms find it difficult obtain financing by traditional models. These enterprises do not fall in the priority list of financial institutions in the funding/loan business. With the use of BTP-SCF all transactions can be executed and completed in a secured manner and also they can be stored using time stamps. Any member node can access any information in the network by generating a query based on its permission levels. Since smart contracts execute and complete financial transactions, the speed and efficiency is enhanced. This also reduces moral disputes if any since everything is computer operations and all the records are stored immutably.

4) Logistics service providers: In the supply chain finance business, logistics service providers act as an auxiliary node. They help the financial institutions by verifying the status of the pledged goods by updating information on the Blockchain platform. This information plays a vital role for financial institutions to make lending decisions any time. Coupling with technology like IoT, institutions can get real time information about the location, weight, status outline with real time live supervision. In order to make the information timely and accurate, BTP-SCF combines blockchain technology with IoT technology. While IoT technology helps in collection of information, the Blockchain technology helps in securely and immutable storing the collected information.

\subsection{The conceptual framework of BTP-SCF}

In the conceptualization of the framework of BTPSCF, the six primary layers. The six layers include:
a. Application, Contract,
b. Incentive, Consensus,
c. Network and
d. Data Layer.

Figure 3 depicts the conceptual framework of BTP-SCF. The traditional models need to be reformed and the supply chain finance business processes are mainly involved in the Consensus, Contract and Application layers. 


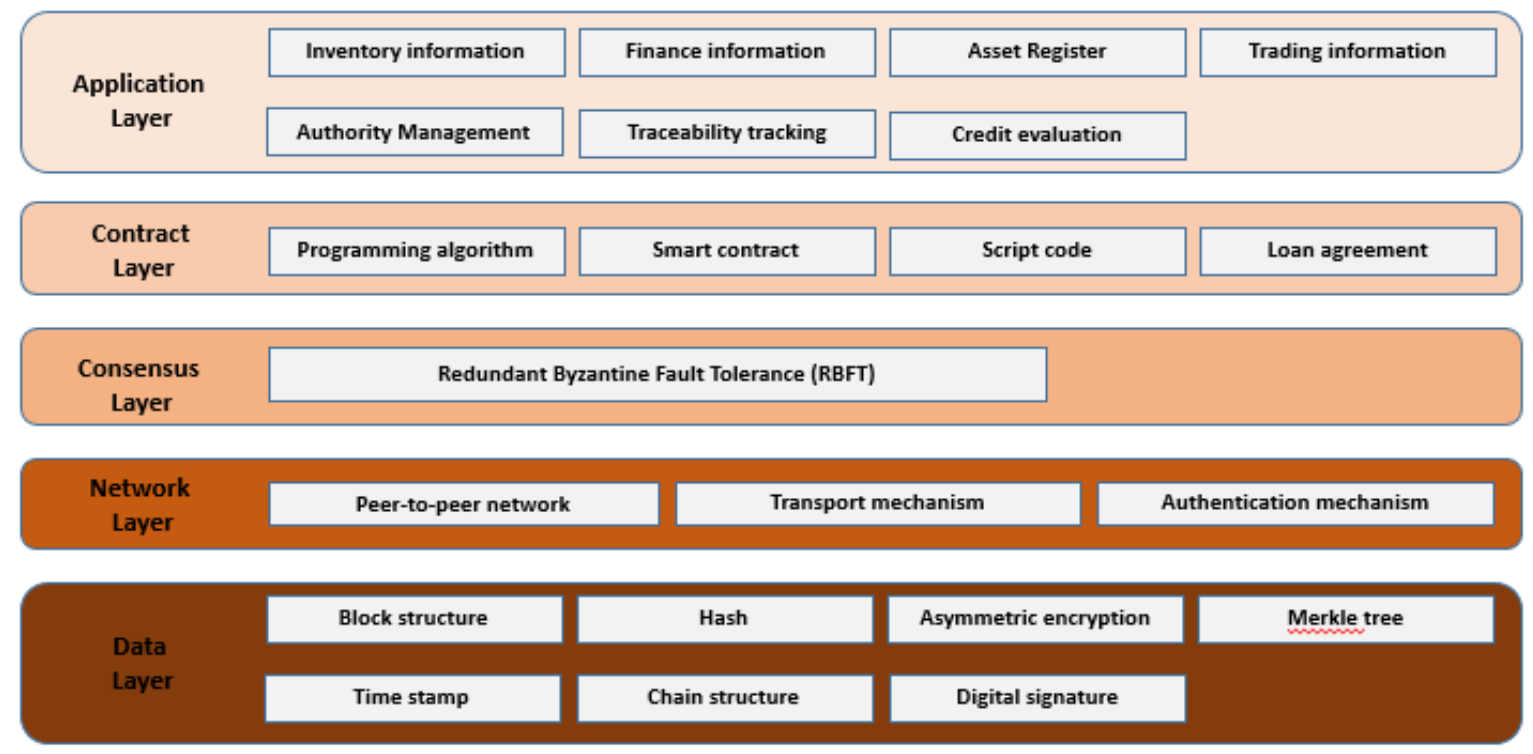

Figure 3: Conceptual framework of BTP-SCF

The data is recorded by mining to the blockchain platform in the Bitcoin system. As a part on incentive mechanism, the miner gets certain amount of Bitcoin as a reward for successfully completing a mining. This type of incentive based operations was necessary for the Bitcoin functioning to be smooth and constraints free. For the application business scenario in supply chain funding, at the nodes, there are more participating companies or enterprises instead of just individual players. The primary intention of the enterprises taking pat on the blockchain platform of accounting is to validate and authenticate the originality of their transactions in their business activities and get them verified by consensus. This will help them obtain loan from financial institutions easily. Since the firms are not participating to get any rewards, the incentive mechanism is not required in this scenario. Thus, this architecture does not incorporate in incentive layer.

Application layer: In a consortium blockchain, the interface that exists between a node and the platform is known as the Application layer. All the business processes that are involved in the supply chain finance regulatory activities and investigation of credit are executed and completed on this layer. The information on financing and transactions, the registrations of assets are all stored onto the blockchain via the application layer. The companies which are seeking loan, can have all their transactional data uploaded onto the blockchain platform using the application layer, which can be later used by financial institutions for evaluation. Based on the referring this information by generating queries, the financial institutions can decide upon whether to sanction loans and what amount of loans to be sanctioned. The logistics service providers update the information on inventory and the real time current status of the goods onto this layer. Regulatory body will provide its service of authority reviewing and settling of disputes if any by interacting with other nodes in the network through the application layer. With the help of digital assets they can trace the flow of information and also keep a track of the transaction of loan seeking enterprises and their loan statuses.

Contract layer: This is the layer where the script code resides and the smart contract is coded. All the programming algorithms which define the working of the blockchain platform reside here. Using the blockchain data, In order to enable the stake holders at nodes to customize smart contracts or host certain decentralized application on the platform, a flexible code system is put in place which allows value transfer by authorizing asset management and data processing. Using program codes all the protocols between each node are defined and programmed. Based on the definitions in the smart contract, the program executes the contract automatically, if all the pre mentioned conditions are satisfied. This prevents moral disputes if any that would occur in traditional scenarios where contracts were executed manually. Refer to Table 1 which shows some functions coded as smart contracts. 


\begin{tabular}{|c|l|}
\hline Function & \multicolumn{1}{|c|}{ Smart Contract code content } \\
\hline Loan & $\begin{array}{l}\text { Has predefined terms and conditions of the loan contract, signed by both the financial } \\
\text { institution(bank) and the enterprise which has taken loan. The smart contracts enables automatic } \\
\text { loan re-payments on the due dates. }\end{array}$ \\
\hline Goods supervision & $\begin{array}{l}\text { loT technology helps capture the real time location and conditions of the goods and uploads it to the } \\
\text { BTP-SCF platform. Based on the defined conditions, an alarm is generated if there are any } \\
\text { discrepancies in the current state of the goods. }\end{array}$ \\
\hline Discount & $\begin{array}{l}\text { Discounts can be automatically granted for customers buying certain defined quantity purchase or } \\
\text { for collaborated customers }\end{array}$ \\
\hline Repurchasing & $\begin{array}{l}\text { In case the company is not able to repay the loan instalment because of its low sales revenue, and } \\
\text { the company has surplus unsold goods in stock, the smart contract can automatically transfer the } \\
\text { ownership of the surplus goods and transfer payments to settle the conditions mentioned in the } \\
\text { contract. }\end{array}$ \\
\hline Tax & Due taxes payments can be automated to the regulatory bodies \\
\hline Transport & $\begin{array}{l}\text { With the help of loT, conditions and real-time can be monitored of the transport of goods. Any } \\
\text { problems during the transport can be easily traced back. Increases visibility of the goods. Goods can } \\
\text { be directly rejected if transport conditions were poor. }\end{array}$ \\
\hline
\end{tabular}

Table 1: Examples of some functions coded in Smart contracts

Consensus Layer: This layer ensures that the consensus mechanism of all the system present on the BTP-SCF platform are effectively executed and completed. Ensures all validations such as Proof-of Stake (POS) and Proof-of-Work (POW) should happen in a decentralized manner. Since dependency of multiple entities, frequent transactions and co-existence are a primary features of supply chain finance business, an hyperchain is required to improve the consensus mechanism. Hence this platform of BTP-SCF should use Redundant Byzantine Fault Tolerance (RBFT) consensus mechanism. In order to facilitate dynamic addition and deletion of nodes as well as enable active recovery RBFT is used. This can handle over ten thousand transactions per second at the same time enhancing the reliability and flexibility of the consensus algorithm.

Network Layer: The transfer of information happens via this layer on the blockchain platform. This is an integral part of a peer-to-peer network and carries out the information transfer protocol at the same time coupling it with the information verification and consensus mechanism. All the member nodes on the blockchain platform form the peer-to-peer network and all of them are treated equal. This enables all the nodes in the network to have a communication and value exchange (peer-to-peer) without the involvement of any enabling institution or a server. This peerto-peer interaction eliminates the possibility of tampered information since, all the node members in the network have a copy of the same ledger and the data on it is immutable. This also reduces the cost of communication among the network members.

Data Layer: The functioning of the data layer in the consortium blockchain is enabled by many computing methodologies, functions and techniques. These are hash function, timestamp technology, digital signature technology, asymmetric encryption, chain structure, Merkle tree and the block. The transactions between the node members are verified by the consensus mechanism and information like transaction value, sanctioned loan, volume and cost of goods, inventory status etc are all stored on multiple blocks. With the help of time stamps they are stored in sequential order of time and hence a chain is formed when these blocks are arranged together. The hash function ensures the privacy of the stored information. The block generates unique and special hash value for all the transaction data that are stored in it. The data is stored in The Merkle tree in such a way, it combines all the has values and generates a unique hash value which is specific to a block. On a consortium blockchain every member at the node as a public and private key each. To ensure the credibility and security of private information there is a need for asymmetric encryption. This is also supported by digital signatures to show consensus and credibility and this is enabled by the private and the public keys. The hash is encrypted using the public key and the information before sending using the asymmetric encryption mechanism. The receiver uses the private key to decrypt it and view the information. Who can view what can be coded accordingly. 
This activity is pictorially represented in Figure 4. The activity of application of digital signature is pictorially represented in Figure 5. Firstly, $\mathrm{P}$, the sender writes the contract, hashes it, encrypts the hash value with Q's public key and sends it to recipient Q. Q, with the help of Q's private key decrypts the hash value. The hash operation on the contract file is simultaneously. By this process, after decrypting and computing, both the hash values are found to be equal and same, the mechanism renders the digital signature as valid.

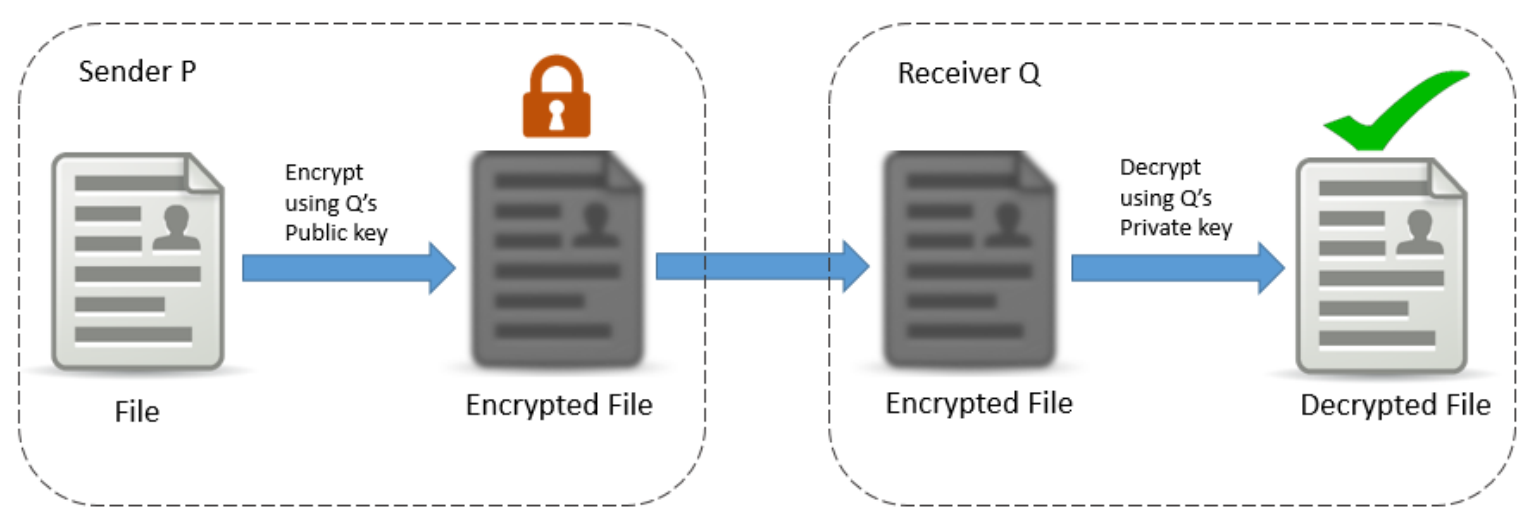

Figure 4: The process of data encryption

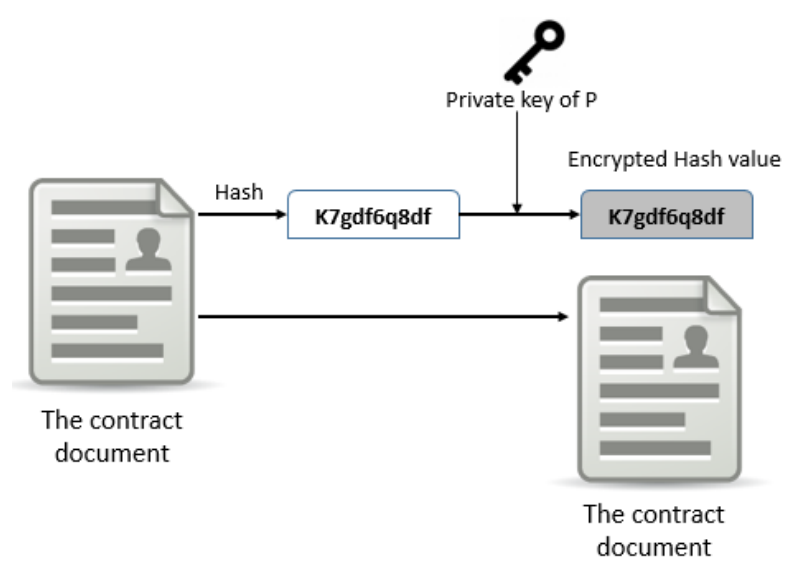

Digital signature process

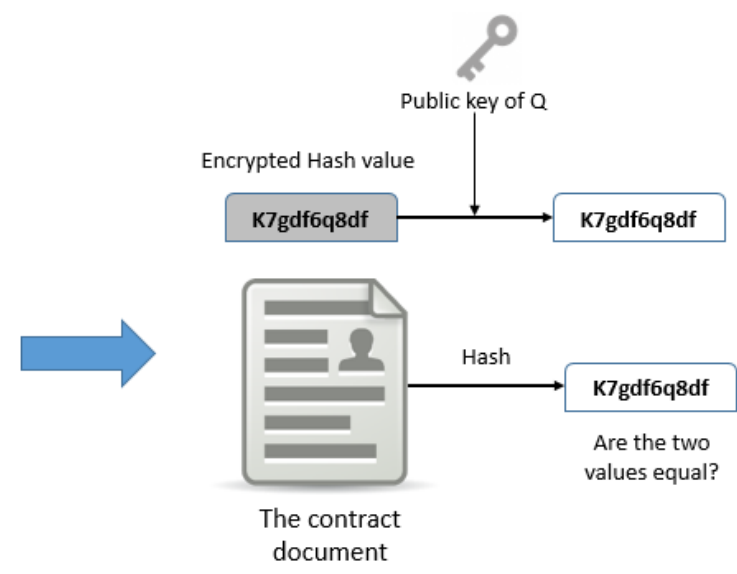

Validation process

Figure 5: Application of Digital Signature and its validation process

\section{BTP-SCF for conducting Supply chain finance business processes}

In the business of supply chain financing, the three main modes we consider for application on the blockchain platform are pre-payment financing, accounts receivable financing and asset pledge financing. Here, let us consider inventory for asset pledging. These activities take place at different stages in the operational activities of the companies. By onboarding on the BTP-SCF platform all the small and medium enterprises can conduct their financial and asset transaction on the blockchain platform and the financial institutions can obtain the necessary information about the enterprises seeking loan from the platform. In this section we will see the three business processes of supply chain financing on the BTP-SCF platform.

\subsection{Prepayment Financing}

Many small and medium enterprises apply for loan on the basis of the potential value of the future inventory. They do this with the relevance of re purchasing from the core companies. For this, once both the parties (financial institution and the company taking loan) reach an agreement and sign a contract, the loan seeking company will keep the goods in Logistic/warehouse service providers recommended by the banks. Here, the bank has the liberty and compulsion to receive the delivery. The company which has taken the loan, is liable to pay the financial institution in installments. Accordingly, the financial institution distributes the goods. If at any point of time the company which has taken loan fails to repay the loan amount and defaults on its terms, the core 
company will repurchase these remaining goods. This entire business process follows principle of signing contracts and selling, supported by feasibility analysis. The proposed process on the blockchain platform BTP-SCF is shown in Figure 6.

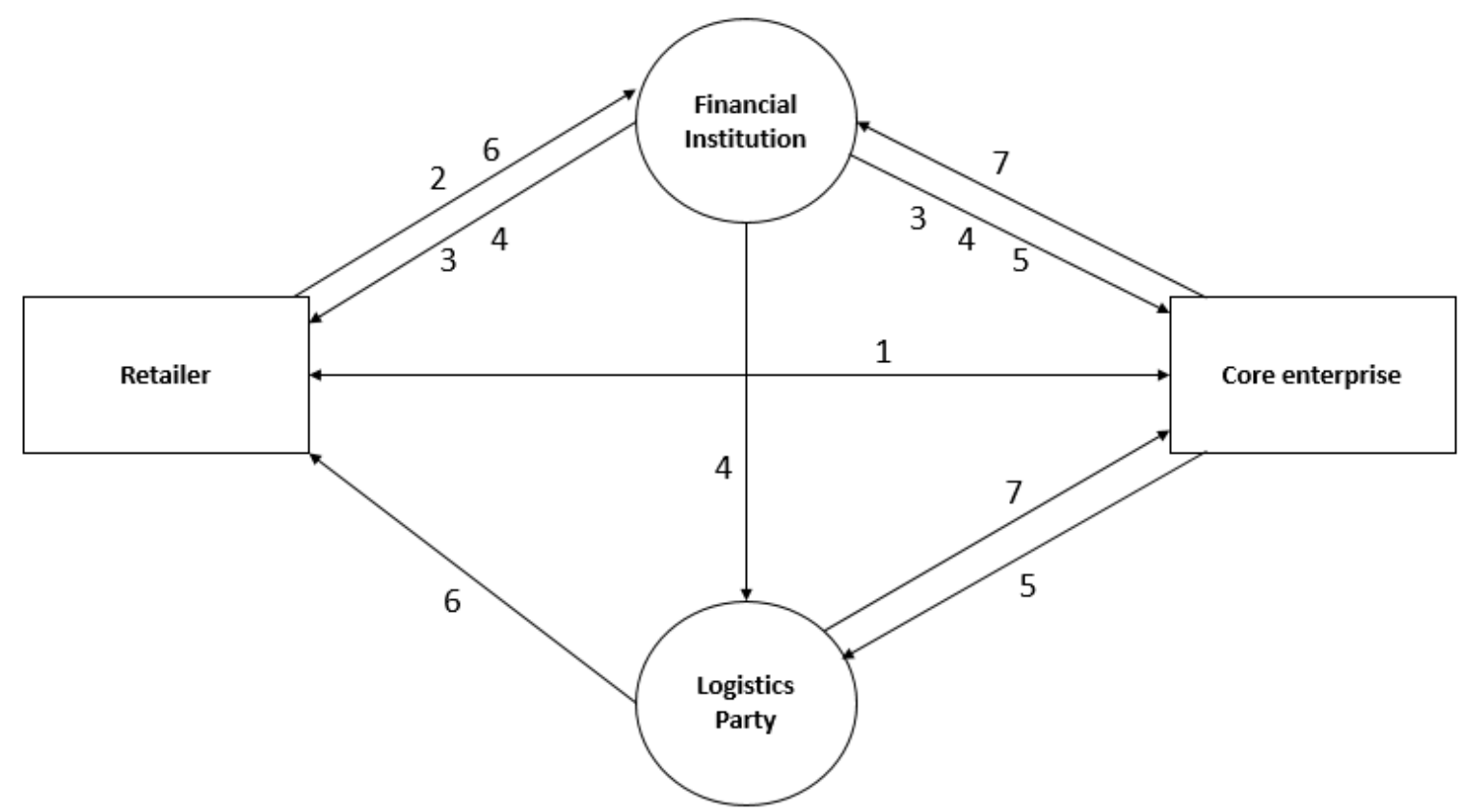

Figure 6: Prepayment Financing on BTP-SCF platform

1. A contract is signed (both online and offline) between the core company and the retailer. Based on the contract terms the platform creates smart contracts. The core company gives a consensus for the retailer in order to pay for the goods, will seek to obtain loan from the financial institutions. On the BTP-SCF platform, the retailer applies for loan.

2. With the help of the data on the BTPSCF platform, the financial institution verifies the contract authenticity of the retailer and evaluates all it transactions and historical operational activities.

3. A regulatory agreement is established between the financial institution and the logistics provider. A loan contract is signed with the retailer and a repurchase contract is signed with the core company. All the contracts are digitally signed and corresponding a smart contract is generated for each on the BTP-SCF platform.

4. Once the acceptance letter is issued by the financial institution the core company sends the goods to be stored to the Logistics party. The bills and receipts generated for each transactions is stored on the BTP-SCF platform.
5. The retailer pays the financial institute in installments the agreed upon amount. On payment he gets the bill of landing and he will get the goods delivered. This cycle is re iterated till all the goods are delivered to the retailers. All the bills generated at every transaction is stored on to the BTP-SCF platform.

6. If the installment is defaulted by the retailer, the smart contract coded for repurchasing is automatically activated. It transfers the payment from core companies account back to the financial institution. The ownership of the assets (goods) are also immediately reversed.

\subsection{Account Receivable financing}

This is system where the suppliers apply for loans to the financial institutions by pledging the Accounts receivable receipts from the core companies as collateral. When the core company makes the payment, a partial payment goes to the financial institution based on agreement and remaining to the supplier. The main constraint in the traditional method is the resource consumption to investigate the credit and transaction history also verifying the authenticity of the existing liable debts. Using smart contracts on the BTP- 
SCF background, this problem can be overcome.

Figure 7 shows this process.

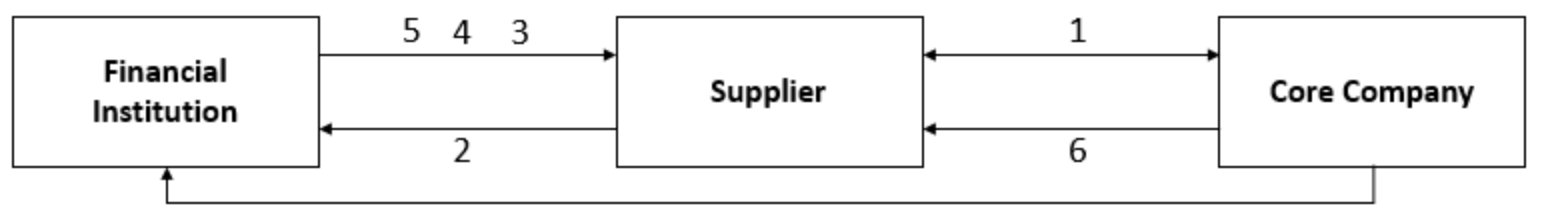

6

Figure 7: Process of account receivable financing on BTP-SCF

1. A contract (both online and offline) of supply is signed between the core company and the upstream supplier. The platform generates the necessary smart contracts according to the involved agreed upon terms and conditions. After every delivery, accounts receivable receipt is generated.

2. With help of the account receivable receipt, the supplier will apply for loan to the financial institution on the BTP-SCF platform.

3. Based on the data of the supplier on the BTP-SCF, the financial institution investigates the loan application by gathering required information and also authenticating the accounts receivables pledged through BTP-SCF. Then the financial institution performs a feasibility analysis regarding the sanction of loan to that supplier.

4. After deciding upon the sanctioned loan amount, tenure and other supporting clauses, the financial institutions sign an agreement to sanction loan amount to the supplier. According to the terms and conditions in the agreement, a smart contract is generated by BTP-SCF.

5. The funds are transferred from the financing institutes to the supplier.

6. After the expiration of the specified time of the accounts recievable, the loan smart contract automatically deducts the stipulated amount from the core company's account to the financial institutions account and the remaining to the suppliers account.

\subsection{Asset pledge financing}

This is a system where some companies keep their assets (here inventory and goods) as collateral to obtain loan from financial institutions. Here a third party player (usually warehouse or a logistics service provider) plays a pivotal role in evaluating and supervising the pledged good or inventory by assisting the financial institutions to conduct the loan sanction and collection operation. Figure 8 shows this

process.

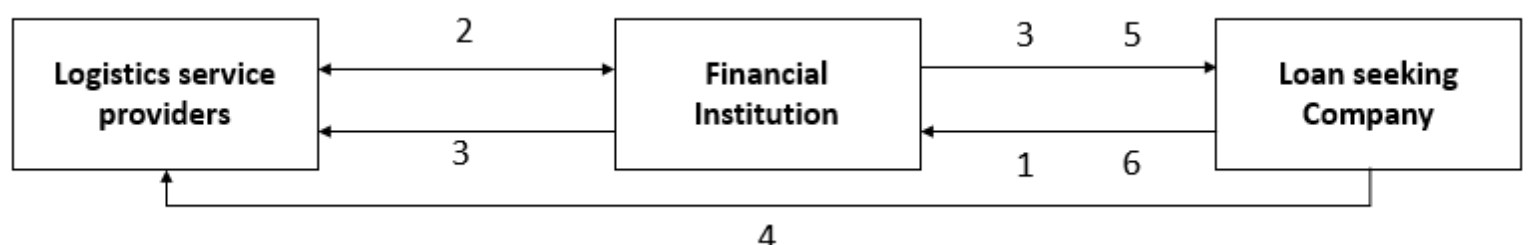

Figure 8: Asset pledge financing process on BTP-SCF

1. A loan seeking company, uploads its asset (inventory or goods data) onto the BTPSCF and applies for loan to the financial institute on BTP-SCF by pledging their assets as collateral.

2. The logistics service provider will evaluate the pledge on behalf of the financial institute and provides a certificate regarding the conditions and location of the goods by uploading relevant information onto the BTP-SCF.
3 . If the information regarding the collateral pledged is verified, authenticated and considered valid, the financial institute will sign the loan agreement with the company and will sanction the loan. It will also sign a supervision agreement or contract with the logistics service provider. Simultaneously, according to the terms and conditions of the contract the BTPSCF generates loan agreement smart contract and a supervision smart contract. 
4. The company will deliver the goods to the logistic service provider where the goods are continuously monitored using IoT technology. This continuously updates information on the BTP-SCF regarding the real-time conditions of the goods. As per contract, the logistic service provider also updates the information on the BTP-SCF. Whenever there is any discrepancy in the conditions of the goods an automatic alarm is triggered on the BTP-SCF.

5. Sanctioned loan amount is transferred to the company by the financial institution.

6. On the agreed upon due dates, the loan smart contract is triggered and it executes the repayment operation by transferring the due amount from the company's account to the financial institution account. Simultaneously, the corresponding ownership of the pledged good is also reversed.

\section{Conclusion}

The research proposed a Blockchain technology solution to overcome the problems face in traditional business processes of supply chain funding. The ease operations for three scenarios of SCF was illustrated on the BTP-SCF platform. The usage of the proposed solution will make the existing process more trusted and secure. It will increase the transparency in the business processes and also reduce the time of transactions. The platform showed that the credit risks and evaluation resources can be split. The block chain technology based SCF solution will enable many financial institutions to develop new business strategies and launch more relevant financial products.

Along with the advantages, there many constraints as well with this technology. The proposed Blockchain technology ensures only the immutability of the information on the blockchain. But it cannot guarantee the truth of the information before it is uploaded to the blockchain. If there is a fraud the source of the data, blockchain cannot take this into account and the entire process fails. As a scope for future work, more research has to be conducted towards analyzing the impact of blockchain son supply chain funding. One should qualitatively and quantitatively study the barriers for adoption of blockchain technology by enterprises and financial institutions and their underlying reasons. A study on what bridges and motivates enterprises to adopt blockchain technology is very necessary.

\section{References}

[1] Akins B W, Chapman J L, Gordon J M (2014). A whole new world: Income tax considerations of the Bitcoin economy. Pittsburgh Tax Review, 12: 25

[2] Álvarez-Díaz N, Herrera-Joancomartí J, Caballero-Gil P (2017). Smart contracts based on blockchain for logistics management. In: Proceedings of the $1 \mathrm{st}$ International Conference on Internet of Things and Machine Learning. Liverpool: ACM, 73

[3] Babich V, Hilary G (2019). Distributed ledgers and operations: What operations management researchers should know about blockchain technology. Georgetown McDonough School of Business Research Paper No. 3131250

[4] Budin M, Eapen A T (1970). Cash generation in business operations: Some simulation models. Journal of Finance, 25(5): 1091-1107 Burkart M, Ellingsen T (2004). In-kind finance: A theory of trade credit.

[5] American Economic Review, 94(3): 569590

[6] Chod J (2017). Inventory, risk shifting, and trade credit. Management Science, 63(10): 3207-3225

[7] Chod J, Trichakis N, Tsoukalas G, Aspegren $\mathrm{H}$, Weber M (2019). On the financing benefits of supply chain transparency and blockchain adoption. Management Science. In press, doi: 10.1287/ mnsc.2019.3434

[8] Fabbri D, Menichini A M C (2016). The commitment problem of secured lending. Journal of Financial Economics, 120(3): 561-584

[9] Foroglou G, Tsilidou A L (2015). Further applications of the blockchain. In: 12th Student Conference on Managerial Science and Technology. Athens 
[10] Haley C W, Higgins R C (1973). Inventory policy and trade credit financing. Management Science, 20(4-part-i): 464-471

[11] Hofmann E, Strewe U M, Bosia N (2017). Supply Chain Finance and Blockchain Technology: The Case of Reverse Securitization. Berlin: Springer

[12] Huang Y X, Liang Z H, Wang Y H, Lu G H (2018). Research on the application of blockchain in supply chain finance. Computer Science and Application, 8(1): 78-88 (in Chinese)

[13] Li X Q, Jiang P, Chen T, Luo X P, Wen Q $Y$ (2020). A survey on the security of blockchain systems. Future Generation Computer Systems, 107: 841-853

[14] Li Z, Wang W M, Liu G, Liu L, He J, Huang G Q (2018). Toward open manufacturing: A cross-enterprises knowledge and services exchange framework based on blockchain and edge computing. Industrial Management \& Data Systems, 118(1): 303-320

[15] Lin I C, Liao T C (2017). A survey of blockchain security issues and challenges. International Journal of Network Security, 19(5): $653-659$

[16] Nakamoto S (2018). Bitcoin: A peer-to-peer electronic cash system.

[17] Available at: bitcoin.org/bitcoin.pdf

[18] Noyes C (2016). BitAV: Fast anti-malware by distributed blockchain consensus and feedforward scanning. Cryptography and Security, Computer Science, Cornell University. Available at: arxiv.org/pdf/ 1601.01405.pdf

[19] Peters G, Panayi E, Chapelle A (2015). Trends in cryptocurrencies and blockchain technologies: A monetary theory and regulation perspec- tive. Journal of Financial Perspectives, 3(3)

[20] Pun H, Swaminathan J M, Hou P (2018). Blockchain adoption for combating deceptive counterfeits. Kenan Institute of Private Enterprise Research Paper No. 18

[21] Saberi S, Kouhizadeh M, Sarkis J, Shen L (2019). Blockchain technology and its relationships to sustainable supply chain manage- ment. International Journal of Production Research, 57(7): 2117-2135

[22] Sharples M, Domingue J (2016). The blockchain and kudos: A distributed system for educational record, reputation and reward. In: European Conference on Technology Enhanced Learning. Adaptive and Adaptable Learning. Cham: Springer, 9891: 490-496 Tang C S, Yang S A, Wu J (2018). Sourcing from suppliers with financial constraints and performance risk. Manufacturing \& Service Operations Management, 20(1): 70-84

[23] Tian F (2016). An agri-food supply chain traceability system for China based on RFID \& blockchain technology. In: 13th International Conference on Service Systems and Service Management (ICSSSM).

[24] Wuttke D A, Blome C, Henke M (2013). Focusing the financial flow of supply chains: An empirical investigation of financial supply chain management. International Journal of Production Economics, 145(2): 773-789

[25] Zamani E, He Y, Phillips M (2018). On the security risks of the blockchain. Journal of Computer Information Systems, 1-12

[26] Zhang Y, Wen J (2015). An IoT electric business model based on the protocol of Bitcoin. In: Proceedings of 18th International Conference on Intelligence in Next Generation Networks. Paris: IEEE, 184- 191

[27] Zheng Z B, Xie S A, Dai H N, Chen X P, Wang H M (2018). Blockchain challenges and opportunities: A survey. International Journal of Web and Grid Services, 14(4): 352-375 